\title{
Plastic behaviour of stepped conical shells under impact loading
}

\author{
J. Lellep \& E. Puman \\ Institute of Mathematics, University of Tartu, Estonia
}

\begin{abstract}
The dynamic plastic behaviour of circular conical shells subjected to lateral distributed loading is investigated. The intensity of the loading is assumed to follow the form of the rectangular impulse. The material of shells is an ideal perfectly plastic material obeying the square yield condition and the associated flow law. For determination of maximal residual deflections an approximate method of mode form motions is developed.

Keywords: plasticity, impact loading, conical shell.
\end{abstract}

\section{Introduction}

The wide practical use of thin walled plates and shells as parts of bodies of vehicles and ship hulls involves the need for knowledge about the behaviour of the shells in extreme conditions and under impact loadings.

Exact solutions, approximate theoretical predictions and experimental verifications of the dynamic plastic response of plates and shells to impulsive and impact loading have been presented by Jones [1], Chakrabarty [2], Kaliszky [3], Stronge and Yu [4]. A lot of attention has been paid to the dynamic plastic response of axisymmetric plates (see Jones [1], Shen and Jones [5], Wang et al. [6]). However, much less information can be found in the literature about inelastic behaviour of shells. The limit analysis of circular conical shells subjected to the distributed lateral loading and of shells loaded by the central rigid boss can be found in the books and papers by Chakrabarty [2], Kaliszky [3] also by Ross [7], Lellep and Puman [8].

In the present paper an approximate method is developed for evaluation of residual deflections of perfectly plastic conical shells of piece wise constant thickness subjected to the impact loading. 


\section{Formulation of the problem}

Let us consider a circular conical shell of piece wise constant thickness (Fig. 1). The shell is simply supported or clamped at the outer edge of radius $R$, whereas the inner edge of radius $a$ is absolutely free. The thickness of the shell wall $h$ is piece wise constant. Let us denote

$$
h=h_{j}
$$

for $r \in\left(a_{j}, a_{j+1}\right)$ where $j=0, \ldots, n$ and $a_{0}=a, a_{n+1}=R, r$ beeing the current radius. Here $h_{j}$ and $a_{j}$ where $j=0, \ldots, n$ stand for given constants.

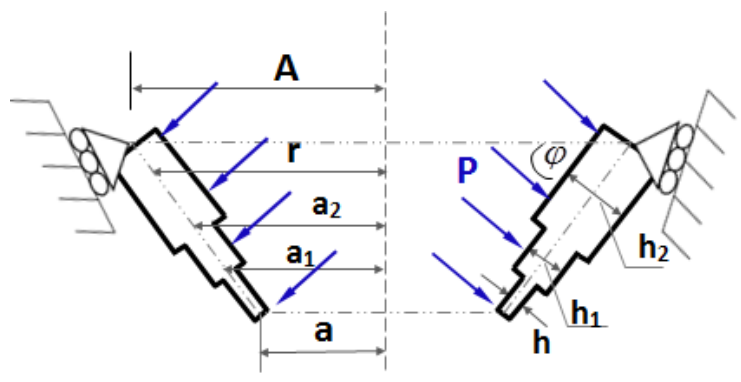

Figure 1: Conical shell subjected the uniformly distributed lateral pressure.

Let the axisymmetric conical shell be subjected to the uniformly distributed lateral pressure loading of intensity $P(t)$ where

$$
P(t)= \begin{cases}P, & t \in\left[0, t_{0}\right] \\ 0, & t>t_{0}\end{cases}
$$

The time interval $\left(0, t_{0}\right)$ is assumed to be a relatively small interval so that during this short interval particles of the shell wall gain certain velocity, the subsequent motion takes place due to the inertia.

The material of the shell is assumed to be an ideal rigid-plastic material. Elastic deformations as well as strain hardening will be neglected in the present paper. It is well known that the concept of a rigid plastic body enables to study the structural behaviour without paying any attention to the stress concentration. This is possible due to the matter that in plastic regions the stress components have limited (finite) values. The rigid plastic model of a material was successfully used by Jones [1], Chakrabarty [2], Kaliszky [3] and others for solving dynamic problems of plastic bodies. It was shown that theoretical predictions compare favourably with experimental data. The exact yield surface is approximated with the squares on planes of bending moments and membrane forces, respectively.

\section{Governing equations and basic assumptions}

Due to the axial symmetry the stress resultants contributing to the internal energy dissipation $\dot{D}_{\imath}$ are the membrane forces $N_{1}, N_{2}$ and bending moments $M_{1}, M_{2}$. 
The influence of the shear stress $Q_{1}$ on the energy dissipation will be neglected in the classical bending theory but it is taken into account in the refined theories. The both approaches will be discussed in the present paper.

The stress resultants must correspond to points lying on the yield surface or being interior points of the surface. Since the exact yield surface corresponding to the Tresca condition has a complicated structure the approximate two-moment limited interaction yield condition will be used in the present paper. This approximation is widely used in the mechanics of inelastic shells [1-4].

The equilibrium conditions of a shell element lead to the equilibrium equations

$$
\begin{gathered}
\frac{d}{d r}\left(r N_{1}\right)-N_{2}=0, \\
\frac{d}{d r}\left[\frac{d}{d r}\left(r M_{1}\right)-M_{2}\right]-\frac{N_{2} \sin \varphi}{\cos ^{2} \varphi}+\frac{r}{\cos ^{2} \varphi}(P-h \bar{\mu} \ddot{W})=0 .
\end{gathered}
$$

Here $\varphi$ is the angle of inclination of the generator of the middle surface of the shell whereas $\bar{\mu} h$ stands for the mass per unit area. The quantity $W$ is the transverse deflection and dots denote the differentiation with respect to time $t$. It is assumed that the displacement $U$ (which is perpendicular with respect to $W$ ) is small and it will be neglected in the present paper.

The strain rate components corresponding to the equilibrium equations eqns (3) can be presented as (here $\varepsilon_{1}, \varepsilon_{2}$ are linear elongations and $\kappa_{1}, \kappa_{2}$ stand for curvatures)

$$
\begin{aligned}
& \dot{\varepsilon}_{1}=\frac{d \dot{U}}{d r} \cos \varphi, \quad \dot{\varepsilon}_{2}=\frac{1}{r}(\dot{U} \cos \varphi+\dot{W} \sin \varphi), \\
& \dot{\kappa_{1}}=-\frac{M_{0}}{N_{0}} \frac{d^{2} \dot{W}}{d r^{2}} \cos ^{2} \varphi, \dot{\kappa}_{2}=-\frac{M_{0}}{N_{0}} \frac{d \dot{W}}{d r} \cos ^{2} \varphi .
\end{aligned}
$$

Here $M_{0}$ and $N_{0}$ stand for the yield moment and the yield force, respectively. Thus $M_{0}=\sigma_{0} h^{2} / 4, N_{0}=\sigma_{0} h, \sigma_{0}$ being the yield stress of the material.

It is reasonable to introduce following non-dimensional quantities

$$
\begin{gathered}
\rho=\frac{r}{R}, \quad \alpha_{j}=\frac{a_{j}}{R}, \quad \gamma_{j}=\frac{h_{j}}{h_{*}}, \quad m_{i}=\frac{M_{i}}{M_{*}}, \quad n_{i}=\frac{N_{i}}{N_{*}}, \quad \varphi_{1}=\frac{\pi}{2}-\varphi, \\
p=\frac{P R}{N_{*} \sin \varphi}, \quad q=\frac{P R}{\sigma_{0} h}, \quad \mu=\frac{\bar{\mu} R h_{*}}{N_{*} \sin \varphi}, \quad k=\frac{M_{*} \cos \varphi}{R N_{*} \sin \varphi} .
\end{gathered}
$$

In (5) $i=1,2 ; j=0, \ldots, n$. Here $h_{*}$ stands for the thickness of the reference shell whereas $M_{*}$ and $N_{*}$ denote the yield moment and yield force for the shell with thickness $h_{*}$. 
Making use of non-dimensional variables eqn (5) one can present the equations of motion as

$$
\begin{aligned}
& \left(\rho n_{1}\right)^{\prime}-n_{2}=0, \\
& k\left[\left(\rho m_{1}\right)^{\prime}-m_{2}\right]^{\prime}-n_{2}+\rho(p-\mu \gamma \ddot{W})=0,
\end{aligned}
$$

where primes denote the differentiation with respect to the variable $\rho$.

\section{Integrating of the equation of motion}

It is assumed that the stress-strain state of the shell corresponds to the horizontal sides $A B$ and $A_{1} B_{1}$ of the yield squares on planes of moments and membrane forces, respectively (Fig. 2). Thus in each region $\left(\alpha_{j}, \alpha_{j+1}\right)$ where $j=0, \ldots, n$ one has $m_{2}=\gamma_{j}^{2}$ and $n_{2}=\gamma_{j}$.

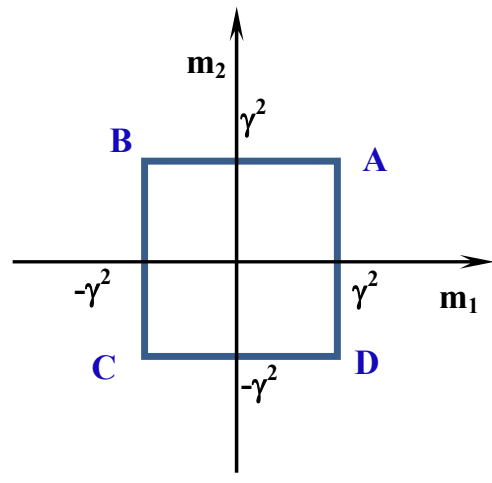

Figure 2: $\quad$ Yield locus.

It is known from the theory of plasticity (see Jones [1]; Chakrabarty [2]; Kaliszky [3]) that the vector with strain rate components must be directed towards the external normal to the yield surface. Applying the gradientality law with respect to the regime $A B$ one can check if $\dot{\kappa}_{1}=0, \dot{\kappa}_{2} \geq 0$. Making use of eqn (4) one can state that $\dot{W}^{\prime \prime}=0$, or

$$
\dot{W}=\dot{w}_{0} \frac{\rho-1}{\alpha-1}
$$

where the boundary requirements

$$
W(\alpha, t)=w_{0}(t), W(1, t)=0
$$

have been taken into account. Here $w_{0}(t)$ stands for an unknown function depending on time only. Evidently, the requirement $\dot{\kappa}_{2} \geq 0$ is fulfilled, if $\dot{w}_{0} \geq 0$.

The flow regime $A_{1} B_{1}$ on the plane of membrane forces is consistent with the associated flow law if $\dot{\varepsilon}_{1}=0, \dot{\varepsilon}_{2} \geq 0$. According to the first requirement one has $\dot{U}=$ const. Thus, approximately one can take $U=0$. 
According to the regimes $A_{1} B_{1}$ and $A B$

$$
n_{2}=\gamma_{j}, m_{2}=\gamma_{j}^{2}
$$

for $\rho \in\left(\alpha_{j}, \alpha_{j+1}\right) ; j=0, \ldots, n$. Inserting eqn (9) in the first equation in eqn (6) one easily obtains after integration

$$
n_{1}=\gamma_{j}+\frac{C_{j}}{\rho}
$$

for $\rho \in\left(\alpha_{j}, \alpha_{j+1}\right)$. In eqn (10) $C_{j}$ stands for an arbitrary constant. Evidently, at the internal edge $n_{1}(\alpha)$ must vanish. Thus $C_{0}=-\gamma_{0} \alpha_{0}$ and

$$
n_{1}=\gamma_{0}-\frac{\gamma_{0} \alpha_{0}}{\rho},
$$

for $\rho \in\left(\alpha_{0}, \alpha_{1}\right)$. Making use of the continuity of the membrane force $n_{1}$ at $\rho=\alpha_{j+1}$ one can write

$$
C_{j+1}=C_{j}+\alpha_{j+1}\left(\gamma_{j}-\gamma_{j+1}\right)
$$

for each $j=0, \ldots, n$. Making use of the recurrent relations eqn (12) one obtains

$$
C_{j}=-\gamma_{0} \alpha_{0}+\sum_{i=1}^{j} \alpha_{i}\left(\gamma_{i-1}-\gamma_{i}\right)
$$

Substituting eqn (13) into eqn (10) results in

$$
n_{1}=\gamma_{j}+\frac{1}{\rho}\left[-\gamma_{0} \alpha_{0}+\sum_{i=1}^{j} \alpha_{i}\left(\gamma_{i-1}-\gamma_{i}\right)\right]
$$

for $\rho \in\left(\alpha_{j}, \alpha_{j+1}\right) ; j=0, \ldots, n$.

It follows from eqn (7) that the acceleration can be presented as

$$
\ddot{W}=\ddot{w}_{0} \frac{\rho-1}{\alpha-1},
$$

for $\rho \in(\alpha, 1)$.

Substituting eqn (9) and eqn (15) in eqn (6) after integration with respect to $\rho$ one obtains

$$
k\left[\left(\rho m_{1}\right)^{\prime}-m_{2}\right]-\gamma_{1} \rho+p \frac{\rho}{2}^{2}-\frac{\mu \gamma_{j} \ddot{w}_{0}}{\alpha-1}\left(\frac{\rho^{3}}{3}-\frac{\rho^{2}}{2}\right)+B_{j}=0,
$$

for $\rho \in\left(\alpha_{j}, \alpha_{j+1}\right) ; j=0, \ldots, n$. Here $B_{j}$ stand for arbitrary constants.

The integration of eqn (16) immediately gives

$$
k\left[\rho m_{1}-\gamma_{j}^{2} \rho\right]-\gamma_{j} \frac{\rho^{2}}{2}+p \frac{\rho^{3}}{6}-\frac{\mu \gamma_{j} \ddot{w}_{0}}{\alpha-1}\left(\frac{\rho^{4}}{12}-\frac{\rho^{3}}{6}\right)+B_{j} \rho+A_{j}=0,
$$

for $\rho \in\left(\alpha_{j}, \alpha_{j+1}\right) ; j=0, \ldots, n$.

The constants of integration $A_{j}, B_{j}(j=0, \ldots, n)$ can be determined making use of the continuity of the shear force $q$ and the bending moment $m_{1}$ at $\rho=\alpha_{j}(j=0, \ldots, n)$. Moreover, $m_{1}\left(\alpha_{0}, t\right)=0, m_{1}(1, t)=0, q\left(\alpha_{0}, t\right)=0$. 
These conditions enable to define the constants of integration and the acceleration $\ddot{w}_{0}$.

\section{Determination of deflections}

It appears that the deformation of the shell takes place during two subsequent stages.

1) The first stage $0 \leq t \leq t_{0}$

During this stage of motion the shell is subjected to the transverse pressure of intensity $P$. The transverse velocity $\dot{W}(\rho, t)$ is defined by eqn (7). It is assumed that the intensity of the loading satisfies the requirement $p \geq p_{0}$ where $p_{0}$ is the lower limit of the pressure when plastic deformations take place. According to this concept $\dot{w}_{0} \equiv 0$, if $p<p_{0}$. It can be shown that $\ddot{w}_{0}=$ const $=\beta_{0}$. Integrating with respect to time one easily obtains

$$
\dot{w}_{0}=\beta_{0} t
$$

and

$$
w_{0}=\frac{\beta_{0}}{2} t^{2},
$$

where the initial conditions $\dot{w}_{0}(0)=0, w_{0}(0)=0$ have taken into account. Thus at the final instant of this stage

$$
w_{0}\left(t_{0}\right)=\frac{\beta_{0}}{2} t_{0}^{2}, \dot{w}_{0}\left(t_{0}\right)=\beta_{0} t_{0} .
$$

2) The second stage $t_{0} \leq t \leq t_{1}$

At the moment $t=t_{0}$ the external pressure is instantaneously removed and the subsequent motion takes place due to inertia. Let us denote $\ddot{w}_{0}=\beta_{1}=$ const for $t \in\left(t_{0}, t_{1}\right)$. After algebraic transformations one has

$$
\begin{gathered}
\beta_{1}=\frac{\alpha-1}{2 \mu T}\left\{\sum _ { i = 1 } ^ { n } ( \gamma _ { i - 1 } - \gamma _ { i } ) \left(2 \alpha_{i}-\alpha_{i}^{2}+2 k \alpha_{i}\left(\gamma_{i}+\gamma_{i+1}\right)-\gamma_{0} \alpha(2+\alpha-\right.\right. \\
\left.\left.\left.2 k \gamma_{0}\right)+\gamma_{n}\left(1+2 k \gamma_{n}\right)\right)\right\},
\end{gathered}
$$

where

$$
T=\gamma_{n}+3 \gamma_{0}\left(\alpha_{0}^{4}-2 \alpha_{0}^{2}\right)+3 \sum_{i=1}^{n}\left(\gamma_{i}-\gamma_{i-1}\right)\left(\alpha_{i}^{4}-2 \alpha_{i}^{2}\right) .
$$

Evidently, the transverse velocity $\dot{w}_{0}(t)$ and the displacement $w_{0}(t)$ at $t=$ $t_{0}$. Accounting for the initial conditions eqn (20) for this stage one can write

$$
\dot{w}_{0}=\beta_{1}\left(t-t_{0}\right)+\beta_{0} t_{0} .
$$

Similarly one obtains

$$
w_{0}=\frac{\beta_{1}}{2}\left(t-t_{0}\right)^{2}+\beta_{0} t_{0}\left(t-t_{0}\right)+\frac{\beta_{0}}{2} t_{0}^{2},
$$

for $t \in\left(t_{0}, t_{1}\right)$. 
At the final instant of time $t=t_{1}$ when the motion of the shell ceases the velocity $\dot{w}_{0}\left(t_{1}\right)$ must vanish. Thus

$$
t_{1}=t_{0}-\frac{\beta_{0} t_{0}}{\beta_{1}} .
$$

Let $w_{1}$ be the maximal residual deflection at the final moment of time. It easily follows from eqns (24), (55) that

$$
w_{1}=\frac{\beta_{0} t_{0}^{2}}{2}\left(1-\frac{\beta_{0}}{\beta_{1}}\right) .
$$

Making use of (7) and (26) one can define the distribution of residual displacements

$$
W\left(\rho, t_{1}\right)=w_{1} \frac{\rho-1}{\alpha-1} .
$$

\section{Discussion}

The results of calculations are presented in Fig. $3-7$ for $k=0,2$ and $\alpha=0,1$.

In Fig. 3 the maximal residual deflections are shown versus the load intensity. Different curves in Fig. 3 correspond to conical shells with different internal radius $a_{0}$. The upper curve corresponds to the shell with $a_{0}=0,1 R$ whereas the lowest one is associated with $a_{0}=0,9 R$. It can be seen from Fig. 3 that the smaller the internal radius the larger the maximal residual deflection, as might be expected. Here the thickness of the shell wall is constant.

Distributions of the bending moment $m_{1}$ for the shell of constant thickness are presented in Fig.4. Different curves in Fig. 4 correspond to different values of the load intensity $p$. The highest curve is obtained for $p=9,4$. Calculations carried out showed that if $p>9,4$ then the solution is not statically admissible.

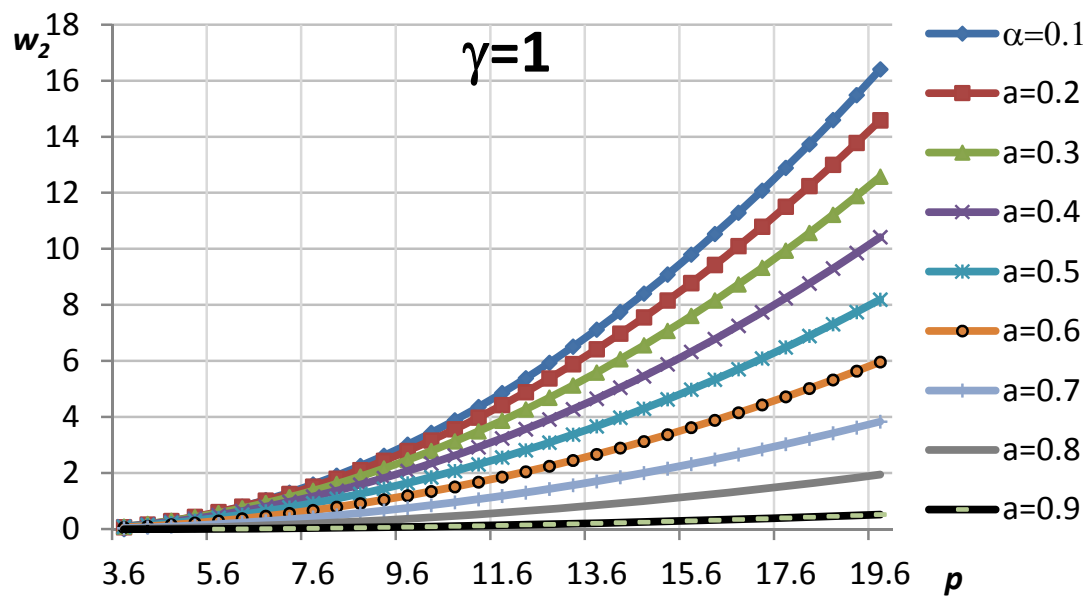

Figure 3: Maximal residual deflections. 


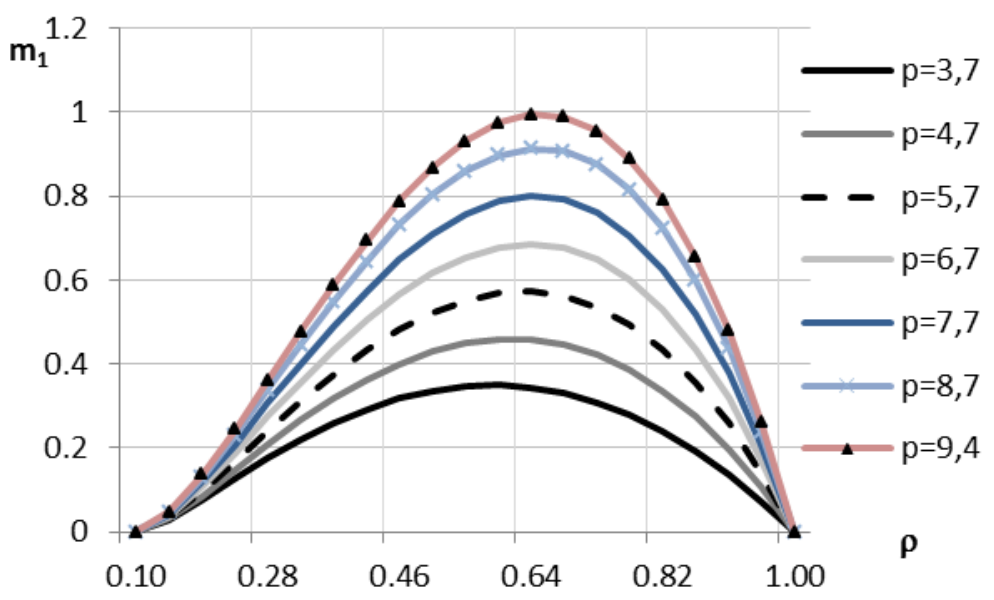

Figure 4: Radial bending moment.

The relationship between the pressure $p_{0}$ and the internal radius $a_{0}$ is depicted in Fig. 5 for different values of the ratio $\gamma$. The upper curve in Fig. 5 corresponds to the shell of constant thickness whereas the lower one is associated with $\gamma=0,1$. It can be seen from Fig. 5 that the quantity $p_{0}$ is not sensitive with respect to $a_{0}$ in the case of small values of $\gamma$.

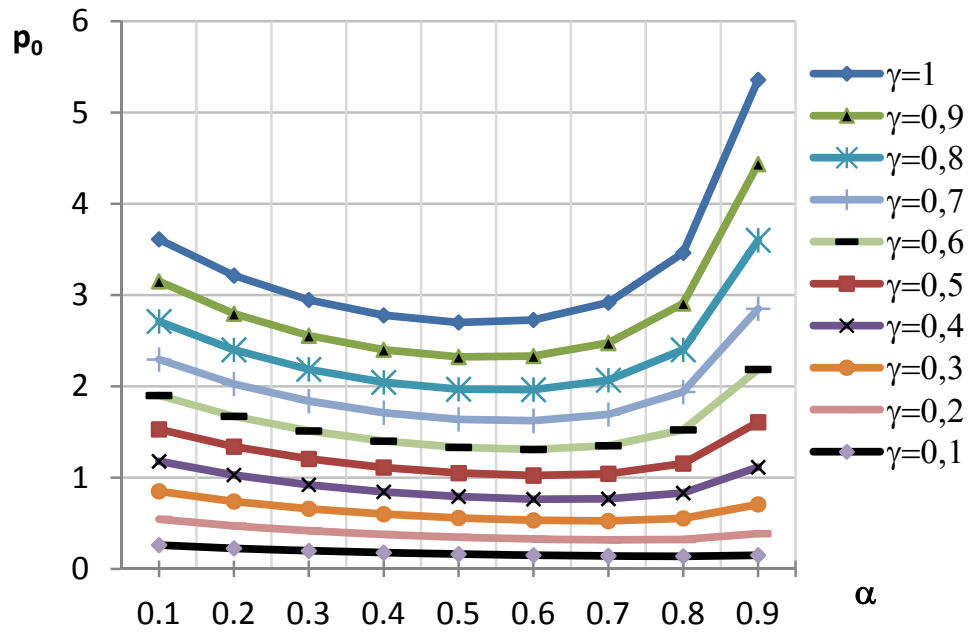

Figure 5: Threshold of the loading.

Maximal residual deflections of stepped shells are presented in Fig. 6. Here the ratio $h_{1} / h_{0}=1,2$. It can be seen from Fig. 6 that when $a_{1}$ increases (the step location moves towards to the supported edge) then maximal residual deflections decrease. This is quite natural because in this case the weight of the shell 
increases. The distributions of the radial bending moment $m_{1}$ are depicted in Fig. 7 for the case $a_{1}=0,35$. Here the ratio of thicknesses $\gamma=1,2$ and different curves correspond to different load intensities.

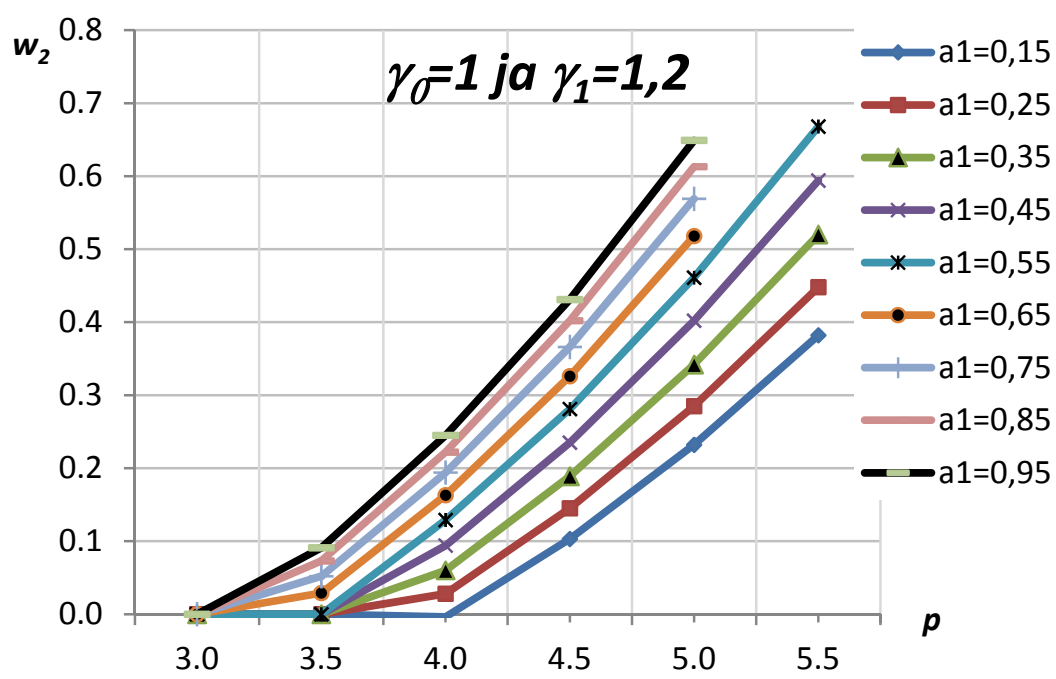

Figure 6: Maximal residual deflections of the stepped shell.

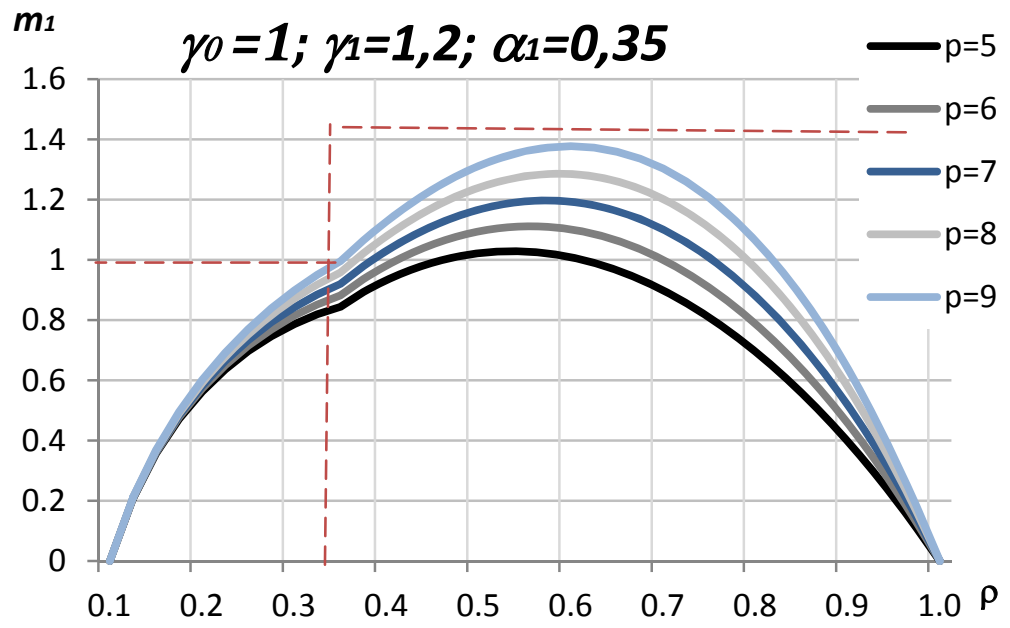

Figure 7: Bending moment $m_{1}$.

In order that the results can be used with confidence, careful comparison and validation with experimental data is needed. However, the experimental database 
is rather limited in the case of conical shells. In Fig. 8 the limit load of the open shell calculated by the current method is compared with the results of Xu et al. [9]. It can be seen from Fig. 8 that the results are quite close to each other.

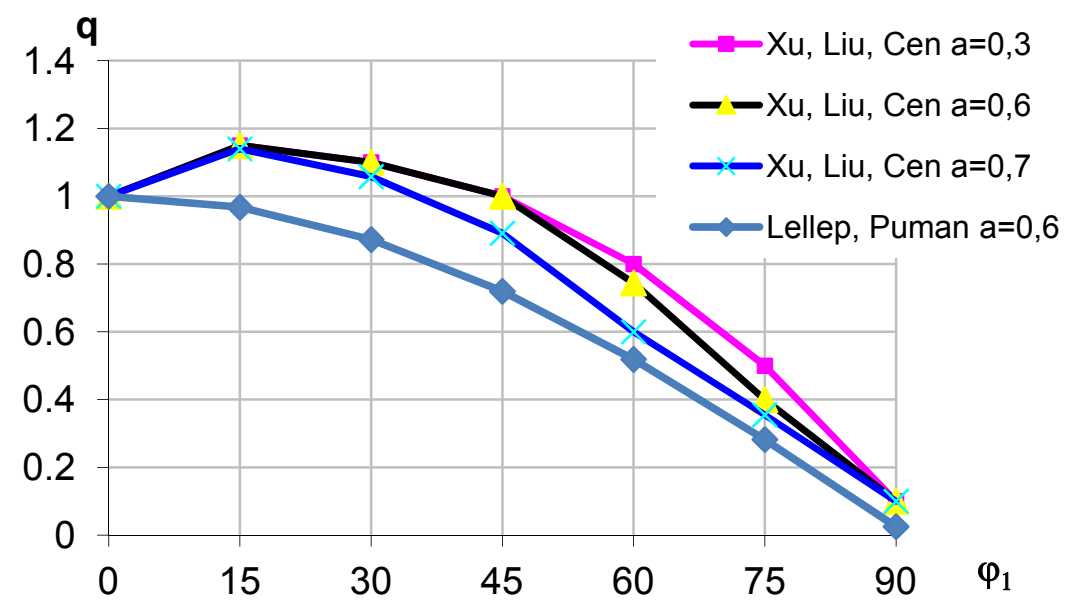

Figure 8: $\quad$ Limit loads.

\section{Concluding remarks}

A method of determination of maximal residual deflections of fully plastic conical shells was developed. The shells under consideration have piece wise constant thickness and are subjected to the rectangular impulse. The material of shells is assumed to be a perfectly plastic one and obeys the square yield condition and associated flow law. The statical admissibility of the solution is assessed numerically.

Calculations carried out showed that maximal residual deflections strongly depend on the ratio of inner and outer radii of the shell.

The solution procedure can be used for approximate evaluation of residual deflections of a shell of variable thickness by suitable choice of $a_{j}$ and $h_{j}(j=$ $0, \ldots, n)$.

\section{Acknowledgement}

The support from the Grant 9110 of ESF "Optimization of structural elements" and from the target financed project SF 0180081S08 "Models of applied mathematics and mechanics" is gratefully acknowledged. 


\section{References}

[1] Jones, N., Structural Impact, CUP: Cambridge, 1989.

[2] Chakrabarty, J., Applied Plasticity, Springer: Berlin, 2000.

[3] Kaliszky, S., Plasticity. Theory and Engineering Applications, Amsterdam: Elsevier, 1989.

[4] Stronge, W. and Yu, T.X., Dynamic Models for Structural Plasticity, Springer: New York, 1993.

[5] Shen, W.Q. and Jones N., Dynamic response and failure of fully clamped circular plates under impulse loading. Int. J. Impact Eng., 13, pp. 259-278, 1993.

[6] Wang, Y., Yu, M., Xiao, Y. and Li, L., Dynamic plastic response of a circular plate based on unified strength theory. Int. J. Impact Eng., 31, pp. 25-40, 2005.

[7] Ross, C.T.F., Pressure Vessels under External Pressure: Statics and Dynamics, Elsevier: London, 1990.

[8] Lellep, J. and Puman, E., Optimization of inelastic conical shells with cracks. Structural and Multidisciplinary Optimization, 33, pp. 189-197, 2007.

[9] Xu, B., Liu, Y. and Cen, Z., Some developments in limit analysis solutions of structures. Metals and Materials, 4(3), pp. 329-335, 1998. 